\title{
Modelo difuso para evaluar la pertinencia de un programa educativo de nivel superior
}

\author{
Sandra Silvia Roblero Aguilar ${ }^{1,2}$, Héctor Rafael Orozco Aguirre², \\ Saturnino Job Morales Escobar ${ }^{2}$ \\ ${ }^{1}$ Instituto Tecnológico de Tlalnepantla, Estado de México, \\ México \\ ${ }^{2}$ Centro Universitario UAEM Valle de México, Estado de México, \\ México
}

ssrauaemex@hotmail.com,hrorozcoa@uaemex.mx,sjob_d@yahoo.com

\begin{abstract}
Resumen. Este artículo tiene como finalidad presentar un modelo, el cual utiliza un sistema de inferencia difuso para evaluar la pertinencia de un programa educativo de nivel superior con respecto al Examen General de Egreso de Licenciatura (EGEL) del Centro Nacional de Evaluación para la Educación Superior (CENEVAL). Los siguientes factores se consideraron para desarrollar el modelo: impacto de cada Unidad de Aprendizaje (UDA) sobre las áreas que evalúa el EGEL, calificación del alumno en cada UDA, apreciación estudiantil promedio en cada UDA, pérdida de conocimiento por años de egreso y ganancia de conocimiento por años de experiencia profesional.
\end{abstract}

Palabras clave: Modelo difuso, pertinencia, EGEL, programa educativo.

\section{Fuzzy Model to Evaluate the Relevance of a Higher Educational Program}

\begin{abstract}
This article presents a model, which uses a fuzzy inference system to evaluate the relevance of a higher educational program in regard to the General Examination for the Degree Exit (EGEL) of the National Center for Higher Education Assessment (CENEVAL). To develop the model the following factors were considered: the impact of each Learning Unit (UDA) on areas EGEL assessment, the score obtained by the student in each UDA, the average student appreciation for each UDA, the loss of knowledge from years of graduation, and the gain of knowledge from years of professional experience.
\end{abstract}

Keywords: Fuzzy model, relevance, EGEL, educational program. 


\section{Introducción}

En México, el Programa Sectorial de Educación 2013-2018, busca lograr como uno de sus objetivos "Fortalecer la calidad y pertinencia de la educación media superior, superior y formación para el trabajo, a fin que contribuyan al desarrollo de México" [1]. Ahora bien, para lograr el aseguramiento de la calidad de los programas y la fortaleza de las Instituciones de Educación Superior (IES), en nuestro país se han impulsado mecanismos, de los cuales sobresalen: las evaluaciones que llevan a cabo los Comités Interinstitucionales para la Evaluación de la Educación Superior (CIEES); las acreditaciones de programas que se efectúan al amparo del Consejo para la Acreditación de la Educación Superior (COPAES); y los Exámenes Generales de Egreso de la Licenciatura (EGEL) del Centro Nacional de Evaluación (CENEVAL).

Dado lo anterior, resulta necesario hacer una evaluación de la relación de los Programas Educativos (PE) con respecto a las áreas que evalúa el EGEL. Tomando como ejemplo al Centro Universitario UAEM Valle de México (CU UAEM VM), perteneciente a la Universidad Autónoma del Estado de México (UAEM), desde 2003 se ha impulsado al EGEL como una modalidad de titulación. Aunque los resultados no han sido los esperados, como se observará más adelante, es conveniente decir que tampoco se han realizado estudios comparativos de los contenidos de los PE con relación a las áreas que evalúa el EGEL. En esta investigación se muestra un modelo basado en la teoría de la lógica difusa y su aplicación, en un análisis de la relación de los contenidos de cualquier PE con respecto al EGEL que le corresponda. Para su validación se tomará como caso de estudio al examen en Ingeniería Computacional (EGEL-ICOMPU) aplicado al PE Ingeniería en Sistemas y Comunicaciones (ISC) versión flexible [2] del CU UAEM VM, que entró en vigor en 2004. El trabajo aquí presentado, se apega a lo establecido en el paradigma cuantitativo, que de acuerdo con Tamayo [3], será mostrado como una investigación de tipo descriptiva.

\section{Descripción del EGEL CENEVAL}

Las calificaciones consideradas en el EGEL-CENEVAL, están expresadas en una escala especial llamada Índice CENEVAL (IC) [4] que va de 700 puntos, calificación más baja; a 1300 puntos, calificación más alta.

En cada una de las áreas evaluadas por el EGEL, se consideran los siguientes tres niveles de desempeño [4]: Aún No Satisfactorio (ANS), Satisfactorio (DS) y Sobresaliente (DSS), en los cuales se clasifica a los sustentantes en función del Testimonio de Desempeño (TD) mostrado, esto es, no satisfactorio si obtiene menos de 1000 puntos, Testimonio de Desempeño Satisfactorio (TDS) si está en el rango de 1000 a 1149 puntos, y Testimonio de Desempeño Sobresaliente (TDSS) en el rango de 1150 a 1300 puntos del IC.

\subsection{Trabajos relacionados con el EGEL}

A continuación, se citan y describen algunos trabajos: 
- Aspectos del EGEL y los resultados de egresados que lo aplicaron durante los años 2001 y 2007 de la carrera de Ingeniería Química del Instituto Tecnológico de Aguascalientes [5].

- Relación entre el promedio académico que obtienen los alumnos de contaduría pública de la Unidad Académica Multidisciplinaria Zona Media, dependiente de la Universidad Autónoma de San Luis Potosí, con el puntaje que logran en el EGEL [6].

- Estadísticas de los resultados que se han obtenido en el EGEL para los egresados de la Universidad de Colima y compartir su experiencia en la elaboración de un examen que se ha aplicado a ocho generaciones de egreso [7].

- Resultados del EGEL de los egresados de los años 2008 a 2010 de la licenciatura en Ciencias de la Educación de la Facultad de Ciencias Humana de la Universidad Autónoma de Baja California [8].

- Mecanismos de mejora de la calidad de los programas de licenciatura que oferta el Instituto Tecnológico de Pachuca, además de presentar estrategias y acciones realizadas en materia de evaluación por medio del EGEL [9].

\section{Lógica difusa}

La lógica difusa está relacionada y fundamentada en la teoría de los conjuntos difusos, en la cual, el grado de pertenencia de un elemento a un conjunto está determinado por una función de pertenencia que puede tomar valores reales comprendidos en el intervalo [0,1], [10], [11], [12]. De esta manera, mientras que en el marco de la lógica clásica un PE tiene pertinencia o no con las áreas que evalúa el EGEL, dándole un valor de 1 si es pertinente y 0 en caso contrario. En la lógica difusa, se obtiene un nivel de cumplimiento de la pertinencia, entre más cercano a cero, será menos pertinente y cuando sea más cercano a 1 será más pertinente.

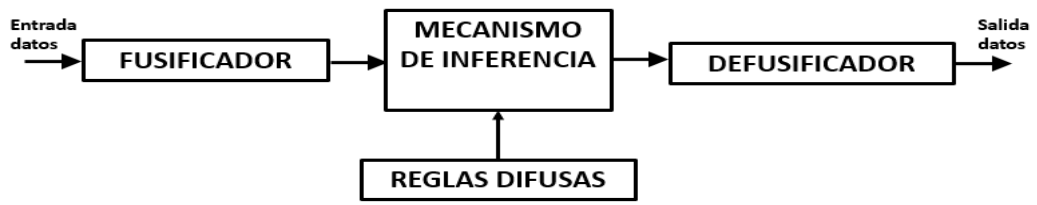

Fig. 1. Sistema de inferencia difuso.

Las etapas que deben cumplirse para el montaje de un sistema de inferencia difuso [12], [13] se muestran en la Fig. 1. Estas se explican a continuación:

- Etapa 1: se basa en un proceso donde las variables tienen un grado de incertidumbre metalingüístico. Es decir, el rango de valores de cada variable puede clasificarse por conjuntos difusos, originando el universo del discurso. Con ello, los valores pasan a un proceso de fusificación que los categoriza en un rango de pertenencia entre 0 y 1 a un conjunto difuso.

- Etapa 2: se proponen reglas lingüísticas conocidas como de inferencia. Con esto, el grado de pertenencia de cada una de las variables se evalúa en un subconjunto de estas reglas. 
- Etapa 3: consiste en determinar los valores óptimos de salida, mediante un mecanismo conocido como defusificación, el cual consiste en pasar el grado de pertenencia, proveniente de la consecuencia de la regla de inferencia activada, a un valor nítido o real, con el fin de obtener un valor cuantificable.

\section{Modelo difuso}

\subsection{Propuesta}

El esquema general del modelo difuso que se propone se puede visualizar en la Fig 2, donde se consideran dos entradas, la primera: son las UDAs de los PEs, a cada una de ellas se les asigna una ponderación, la cual es determinada por el coordinador de carrera, con respecto a los contenidos de cada UDA y la relación con las áreas que evalúa el EGEL, esta asignación, representa la relevancia o grado de impacto del conocimiento en cada una de las áreas. Con esta base y tomando en consideración las calificaciones de las UDAs del sustentante durante su trayectoria académica y la apreciación estudiantil (evaluación realizada por el alumno de la actividad del docente en cada UDA), se realiza un cálculo de estimación simple; la segunda entrada es el histórico de resultados del EGEL, este histórico permite calcular el factor de impacto por área, dependiendo de la pérdida o ganancia de conocimiento del sustentante en cada área con base en los años de experiencia laboral y tiempo de egreso, de esta asignación se tiene como resultado la ponderación acumulada por área, con lo anterior se puede determinar el cálculo de estimación por egreso y por experiencia, lo cual pondera el nivel de impacto de los contenidos de las UDAs por área.

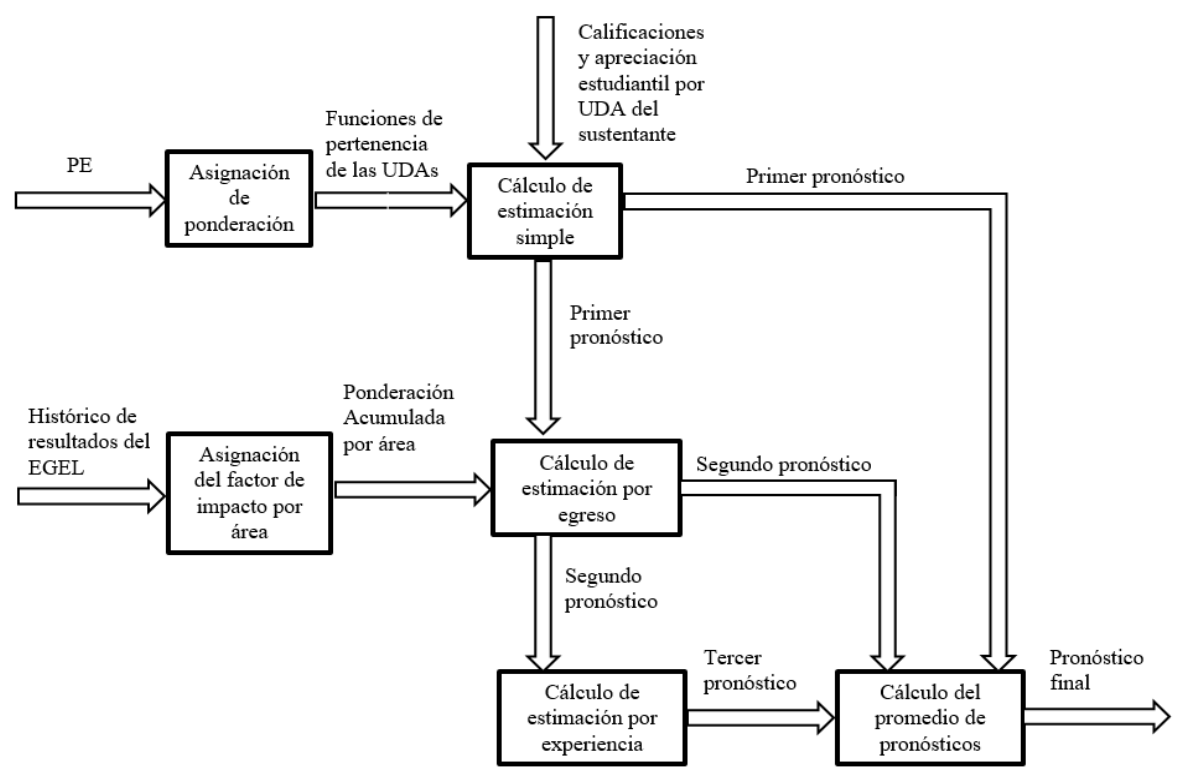

Fig. 2. Esquema general del modelo difuso. 


\subsection{Análisis del PE}

El modelo propuesto consta de lo siguiente:

A) El conjunto de UDAs de un PE, que a partir de este momento será el universo del discurso, está determinado de manera extensional por:

$$
\mu=\left\{\mathrm{UDA}_{1}, \mathrm{UDA}_{2}, \mathrm{UDA}_{3}, \ldots, \mathrm{UDA}_{\mathrm{n}}\right\} .
$$

A cada $\boldsymbol{U D A}$ se le asigna una ponderación con respecto a sus contenidos y la relación con cada una de las áreas del EGEL, que se puede definir como: $\mathrm{P}=$ "tener membresía para cada una de las áreas correspondientes del EGEL". Se define P como el subconjunto de UDAs con membresía a dichas áreas, esta ponderación representa la relevancia o grado de impacto del conocimiento en cada una de las áreas que evalúa el EGEL.

Al finalizar de ponderar a cada UDA con respecto a lo que evalúa el EGEL, se aplica la siguiente ecuación para cada área:

$$
\mathrm{VPA}=\Sigma(\mathrm{PUDA})
$$

donde $\boldsymbol{V P A}$ : valor de ponderación acumulada por área,

$\boldsymbol{P U D A}$ : ponderación asignada a cada UDA en esa área.

El resultado de la aplicación de esta ecuación proporciona la ponderación acumulada por área.

Por otra parte, inicialmente se consideraron 2 factores de impacto, que determinan si un sustentante alcanza o no un TD en el EGEL, estos factores son:

1. La calificación obtenida por el sustentante en cada una de la UDAs en su trayectoria académica.

2. La calificación proporcionada por el alumno a los docentes de cada UDA en el proceso de evaluación docente.

Con los dos primeros factores se obtiene la siguiente estimación (representa el primer pronóstico del esquema general del modelo difuso):

- Estimación Simple por Área $(\boldsymbol{E S A})$ : en esta se considera el valor máximo de $\boldsymbol{V P A}$ y los rangos de puntuación del IC, tal y como se muestra en la siguiente ecuación:

$$
\mathrm{ESA}=\frac{\mathrm{PPA}}{\operatorname{Max}(\mathrm{VPA})} * 6+700
$$

donde $\boldsymbol{P P A}$ es el Puntaje de Ponderación por Área, la cual se obtiene de la siguiente ecuación:

$$
\mathrm{PPA}=\sum((\mathrm{CUDA} * \mathrm{~F} 1+\mathrm{EDO} * \mathrm{~F} 2) * \mathrm{PUDA})
$$

donde:

$\boldsymbol{C U D A}$ es la calificación de la UDA. 
$\boldsymbol{E D O}$ es la evaluación promedio del docente asignado por los estudiantes en esa UDA.

F1 es el factor de impacto de la calificación de la UDA, expresado en porcentaje.

$\boldsymbol{F} \mathbf{2}$ es el factor de impacto de la calificación del docente, expresado en porcentaje.

Los factores de impacto son determinados heurísticamente por el coordinador del PE.

B) Con base en los resultados obtenidos por los sustentantes, se realizó un ajuste a posteriori en el modelo y se agregan dos nuevos factores de impacto: el tiempo de egreso y los años de experiencia profesional, con lo anterior se generan dos estimaciones más, descritos como sigue:

- Estimación Por años de Egreso (EPE): implica el conocimiento que el sustentante ha perdido por el tiempo que tiene de egresado, este factor, definido como Factor de Pérdida $(\boldsymbol{F P})$, debe de calcularse tomando en cuenta tres grados de pérdida, determinados como alto, medio y bajo. Estos grados están relacionados con los puntajes mínimos requeridos esperados en la ecuación 3.

Tabla 1. Cálculo del $\boldsymbol{F P}$ por años de egreso.

\begin{tabular}{|c|c|c|c|c|}
\hline $\begin{array}{l}\text { Grado de } \\
\text { pérdida }\end{array}$ & Valor mínimo requerido & $\begin{array}{c}\text { Puntaje } \\
\text { mínimo } \\
\text { requerido en } \\
E S A\end{array}$ & $\begin{array}{l}\text { Tiempo de } \\
\text { egreso }\end{array}$ & $\begin{array}{c}\text { TD } \\
\text { esperado }\end{array}$ \\
\hline $\boldsymbol{F} \boldsymbol{P}_{\text {Alto }}$ & $\begin{array}{c}150 * \operatorname{Max}(\boldsymbol{V P A}) \\
6\end{array}$ & 850 & $>2$ años & ANS \\
\hline $\boldsymbol{F} \boldsymbol{P}_{\text {Medio }}$ & $\begin{array}{c}300 * \operatorname{Max}(\boldsymbol{V P A}) \\
6\end{array}$ & 1000 & $\begin{array}{c}>=1 \mathrm{y}<= \\
2 \text { años }\end{array}$ & TDS \\
\hline $\boldsymbol{F} \boldsymbol{P}_{\text {Bajo }}$ & $375 * \operatorname{Max}(\boldsymbol{V P A})$ & 1075 & $<1$ año & TDS \\
\hline
\end{tabular}

El $\boldsymbol{F P}$ está asociado al tiempo de egreso del sustentante y al puntaje mínimo requerido en la Estimación Simple por Área.

Al aplicar el $\boldsymbol{F P}$ se puede obtener la siguiente ecuación $\boldsymbol{E P E}$ :

$$
\mathrm{EPE}=\frac{\left(\mathrm{PPA} *\left(\frac{\mathrm{FP}}{\mathrm{VPA} * 100}\right)\right)}{\operatorname{Max}(\mathrm{VPA})} * 6+700 .
$$

- Estimación por Años de Experiencia $(\boldsymbol{E A E})$ : la que el egresado tenga en alguna de las áreas evaluadas por el EGEL. En este factor, se toma en cuenta la experiencia que el sustentante pudiese haber obtenido en cada una de las áreas del EGEL, en función de la o las actividades desempeñadas en el ámbito profesional. Para este factor se considera el resultado de $\boldsymbol{V P A}$ obtenido en cada una de las áreas del EGEL y el $\boldsymbol{F P}$, es decir, el valor mínimo requerido definidos en la Tabla 1, este factor también se da en tres grados: alto, medio y bajo. Con lo anterior se obtiene la Tabla 2. 
Modelo difuso para evaluar la pertinencia de un programa educativo de nivel superior

Tabla 2. Cálculo del factor de ganancia.

\begin{tabular}{cccc}
\hline $\begin{array}{c}\text { Grado de } \\
\text { ganancia }\end{array}$ & Ganancia & $\begin{array}{c}\text { Tiempo de } \\
\text { experiencia }\end{array}$ & $\begin{array}{c}\text { TD } \\
\text { esperado }\end{array}$ \\
\hline $\boldsymbol{F} \boldsymbol{G}_{\text {Alto }}$ & $\boldsymbol{V P A} * 100$ de cada área & $>2$ años & TDSS \\
$\boldsymbol{F G}_{\text {Medio }}$ & $\boldsymbol{F P} \boldsymbol{P}_{\text {Bajo }}$ & $>100$ de cada área & años \\
& $\boldsymbol{F P} \boldsymbol{P}_{\text {Medio }}$ & TDS \\
$\boldsymbol{F} \boldsymbol{G}_{\text {Bajo }}$ & $\boldsymbol{V P A} * 100$ de cada área & $<1$ año & ANS \\
\hline
\end{tabular}

Al aplicar este Factor de Ganancia $(\boldsymbol{F G})$ por años de experiencia, se tiene como resultado la siguiente expresión:

$$
\mathrm{EAE}=\frac{\mathrm{PPA}+((\mathrm{VPA} * 100) * \mathrm{FG})}{\operatorname{Max}(\mathrm{VPA})} * 6+700
$$

Considerando lo anterior, el siguiente paso es calcular el promedio de las tres estimaciones y con ello se obtiene la siguiente ecuación:

$$
\mathrm{PAE}=\frac{\mathrm{ESA}+\mathrm{EPE}+\mathrm{EAE}}{3},
$$

donde $\boldsymbol{P} \boldsymbol{A} \boldsymbol{E}$ es el pronóstico del área del EGEL.

La ecuación 7 se debe realizar para cada una de las áreas del EGEL.

\subsection{Definición de las reglas difusas}

Fuzzy Control Language (FCL) [14], es el lenguaje para definir las reglas del sistema de inferencia difuso que se utiliza en las clases de Java del paquete jFuzzyLogic. FCL es un estándar incluido en el IEC (International Electrotechnical Commission).

Para poder escribir las reglas difusas se consideraron las áreas que evalúa el EGEL y los criterios establecidos para el otorgamiento del TD.

Considerando que no importa el orden de las áreas, se aplica la fórmula de combinación, con la finalidad de establecer el número de reglas requeridas.

$$
\mathrm{C}_{\mathrm{k}}^{\mathrm{n}}=\frac{\mathrm{n} !}{\mathrm{k} !(\mathrm{n}-\mathrm{k}) !}
$$

donde:

$\boldsymbol{n}$ es número de áreas que se evalúan para un PE.

$\boldsymbol{k}$ variables de entrada (número de criterios establecidos para el otorgamiento del TD).

Las reglas del sistema de inferencia difuso, definidas para obtener el TD tienen la forma siguiente: 
- Reglas difusas para TDSS

- RULEj: IF (áreai IS ds OR áreai IS dss) AND

- (áreai+1 IS ds OR áreai+1 IS dss) AND

- ...

- (área $a_{i+n-1}$ IS ds AND áreai+n-1 IS dss) THEN

- td IS dss;

- Reglas difusas para TDS

- RULE ${ }_{j+k}$ : IF (áreai IS ds OR áreai IS dss) AND

- (áreai+1 IS ds OR áreai+1 IS dss) AND

- ...

○ (área $a_{i+n-1}$ IS ds OR área $a_{i+n-1}$ IS dss) THEN

- td IS ds;

- Reglas difusas para ANS

- RULE $_{j+k+1}$ IF (áreai IS ans AND áreai+1 IS ans) THEN

- td IS ans;

Se definen RULE $\mathrm{j}_{\text {, para }} 1<=\mathrm{j}<=\mathrm{m}$ y área $\mathrm{a}_{\mathrm{i}}$, para $1<=\mathrm{i}<=\mathrm{n}$.

Para $1<=\mathrm{k}<=4|6| 10$.

Para $1<=1<=6 \mid 10$.

Donde $\boldsymbol{m}$ corresponde al número total de reglas formadas para un PE y $\boldsymbol{n}$ el número de áreas que se evalúan para un PE.

\subsection{Fusificación y defusificación}

La fusificación y defusificación se aplica a cada una de las áreas de evaluación, considerando la distribución del IC.

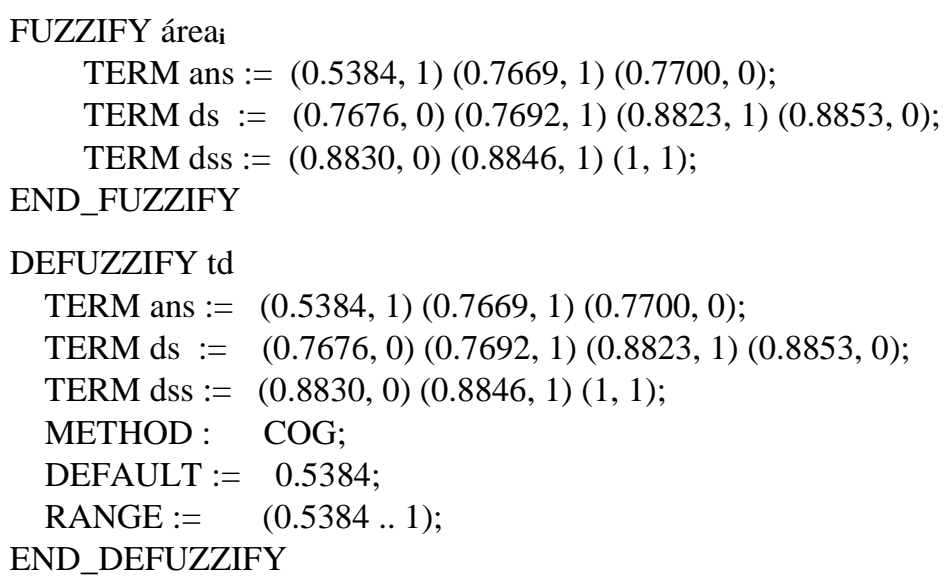

En la Tabla 3, se muestra el mapeo del IC con respecto a los niveles de desempeño del EGEL para la defusificación, la cual se obtiene aplicando una regla de tres, donde 1300 es el $100 \%$. 
Modelo difuso para evaluar la pertinencia de un programa educativo de nivel superior

Table 3. Mapeo del IC para la defusificaciòn.

\begin{tabular}{ccccc}
\hline $\begin{array}{c}\text { Niveles de } \\
\text { Desempeño }\end{array}$ & & Puntos del IC/valor de mapeo & \\
\hline ANS & $700 / 0.5384$ & $997 / 0.7669$ & $1001 / 0.7700$ & \\
DS & $998 / 0.7676$ & $1000 / 0.7692$ & $1147 / 0.8823$ & $1151 / 0.8853$ \\
DSS & $1148 / 0.8830$ & $1150 / 0.8846$ & $1300 / 1$ & \\
\hline
\end{tabular}

Es importante mencionar que para la defusificación se usa el centro de gravedad (COG), lo cual implica que el valor a obtener para el TD se ubica en el centro del rango de pertenencia.

\section{Caso de estudio}

\subsection{Descripción del EGEL-ICOMPU y sus resultados en el PE DE ISC}

En el EGEL-ICOMPU [4], aplicado a egresados de ISC, para que el sustentante obtenga un TDS: de las cinco áreas a evaluar, al menos debe tener cuatro áreas con DS o DSS y para obtener el TDSS: al menos en dos debe alcanzar DSS y las restantes con DS. Las áreas del EGEL-ICOMPU son las siguientes:

- Selección de Sistemas Computacionales para Aplicaciones Específicas (SSCAE).

- Nuevas Tecnologías para la implementación de Sistemas de Cómputo (NTISC).

- Desarrollo de Hardware y Software Asociado para Aplicaciones Específicas (DHSAAE).

- Adaptación de Hardware y/o Software para Aplicaciones Específicas (AHSAE).

- Redes de Cómputo para Necesidades Específicas (RCNE).

Al hacer un análisis de los resultados de sustentantes del EGEL-ICOMPU a nivel nacional, CU UAEM VM y PE ISC entre 2013 y 2015, se tiene la Tabla 4.

Tabla 4. Comparativo de resultados del EGEL-ICOMPU.

\begin{tabular}{ccc}
\hline & ANS & TDS/TDSS \\
\hline Nacional & $50 \%$ & $50 \%$ \\
CU UAEM VM & $46 \%$ & $54 \%$ \\
ISC & $54 \%$ & $46 \%$
\end{tabular}

Como se puede observar en la Tabla 4, a nivel nacional el 50\% de los sustentantes logran obtener un TD aprobatorio (TDS o TDSS), mientras que el mismo porcentaje no lo obtiene (ANS). En el caso del CU UAEM VM, el 54\% de sus sustentantes aprueba y para la carrera de ISC sólo el $46 \%$ logra este resultado. Lo anterior, requiere de una atención inmediata para que se puedan establecer mecanismos que conlleven a que un 
mayor número de sustentantes logren alcanzar un TD, aunado a esto, si se considera que en el CU UAEM VM, el EGEL es una de las modalidades de titulación, sus resultados impactan directamente en el índice de titulación.

Considerando que el PE de ISC consta de 53 UDAs, se tiene como resultado la Tabla 5, en donde se muestra la distribución de las ponderaciones de cada UDA por área.

Al finalizar se pondera a cada UDA con respecto a lo que evalúa el EGEL, se aplica la ecuación 2.

En el resultado de la aplicación, se observa cuáles de la UDAs tienen mayor impacto en el EGEL-ICOMPU con respecto al PE de ISC, en la Tabla 6, se muestra el VPA y el porcentaje que representa.

Aplicando las ecuaciones 4 y 5, se tienen como valores: 275 para un $\boldsymbol{F P}_{\text {Alto }}, 550$ para un $\boldsymbol{P F}_{\text {Medio y }} 687.5$ para un $\boldsymbol{F P}_{\text {Bajo. Es importante mencionar que los valores }}$ determinados por el coordinador de ISC para las variables $\boldsymbol{F 1}$ y $\boldsymbol{F} 2$ fueron 90 y $10 \%$ respectivamente, lo anterior indica que la calificación obtenida en cada UDA tiene mayor impacto en el resultado en comparación a la apreciación estudiantil.

Tabla 5. Distribución de ponderación con respecto a las áreas del EGEL-ICOMP.

\begin{tabular}{|c|c|c|c|c|c|c|}
\hline No. & NOMBRE_UDA & SSCAE & NTISC & DHSAAE & AHSAE & RCNE \\
\hline 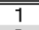 & ALGEBRA LINEAL & 0.2 & $\overline{0.2}$ & 0.2 & 0.2 & 0.2 \\
\hline 2 & ALGEBRA Y GEOMETRIA ANALITICA & 0.2 & 0.2 & 0.2 & 0.2 & 0.2 \\
\hline 3 & CALCULODIFERENCIAL E INTEGRAL & 0.2 & 0.2 & 0.2 & 0.2 & 0.2 \\
\hline 4 & CALCULO VECTORIAL & 0.2 & 0.2 & 0.2 & 0.2 & 0.2 \\
\hline 5 & ECUACIONES DIFERENCIALES & 0.2 & 0.2 & 0.2 & 0.2 & 0.2 \\
\hline 6 & ELECTROMAGNETISMO & 0.2 & 0.2 & 0.2 & 0.2 & 0.2 \\
\hline 7 & ESTATICA Y DINAMICA & 0.2 & 0.2 & 0.2 & 0.2 & 0.2 \\
\hline 8 & INGLESC1 & 0.2 & 0.2 & 0.2 & 0.2 & 0.2 \\
\hline 9 & INGLES C2 & 0.2 & 0.2 & 0.2 & 0.2 & 0.2 \\
\hline 10 & INTRODUCCION A LA COMPUTACION & 0.2 & 0.2 & 0.2 & 0.2 & 0.2 \\
\hline 11 & PRIOBABILIDAD Y ESTADISTICA PARA INGENIEROS & 0.2 & 0.2 & 0.2 & 0.2 & 0.2 \\
\hline 12 & QUIMICA & 0.2 & 0.2 & 0.2 & 0.2 & 0.2 \\
\hline 13 & ADMINNISTRACION & 0.2 & 0.2 & 0.2 & 0.2 & 0.2 \\
\hline 14 & CONTABILIDAD & 0.2 & 0.2 & 0.2 & 0.2 & 0.2 \\
\hline 15 & ECOLOGIA, ETICA Y NORMATIVIDAD & 0.2 & 0.2 & 0.2 & 0.2 & 0.2 \\
\hline 16 & TECNICAS DE COMUNICACIÓN & 0.2 & 0.2 & 0.2 & 0.2 & 0.2 \\
\hline 17 & ALGORITMOSYESTRUCTURA DE DATOS & 0.4 & 0.3 & 0.1 & 0.2 & 0 \\
\hline 18 & ARQUITECTURA DE COMPUTADORAS & 0.3 & 0.2 & 0.3 & 0.2 & 0 \\
\hline 19 & BASESDE DATOS & 0.5 & 0.3 & 0 & 0.2 & 0 \\
\hline 20 & CIRCUITOS ELECTRICOS & 0.1 & 0.1 & 0.4 & 0 & 0.4 \\
\hline 21 & DESARROLLODE PROYECTOS & 0.1 & 0.3 & 0.1 & 0.3 & 0.2 \\
\hline 22 & ELECTRONICA ANALOGICA & 0.1 & 0.1 & 0.4 & 0 & 0.4 \\
\hline 23 & FORMULACIONY EVALUACION DE PROYECTOS & 0.3 & 0.2 & 0.1 & 0.3 & 0.1 \\
\hline 24 & FUNDAMENTOS DE BASES DE DATOS & 0.4 & 0.3 & 0 & 0.3 & 0 \\
\hline 25 & FUNDAMENTOSDE PROGRAMACION & 0.3 & 0.3 & 0.1 & 0.3 & 0 \\
\hline 26 & INGENIERIA DE SOFTWAARE & 0.4 & 0.5 & 0 & 0.1 & 0 \\
\hline 27 & INTRODUCCION A LA INGENIERIA & 0.2 & 0.2 & 0.2 & 0.2 & 0.2 \\
\hline 28 & INVESTIGACION DE OPERACIONES & 0.2 & 0.2 & 0.2 & 0.2 & 0.2 \\
\hline 29 & LENGUA.JES DE BA.JONIVEL & 0 & 0.3 & 0.3 & 0.2 & 0.2 \\
\hline 30 & LENGUA.JES FORMALES Y AUTOMATAS & 0.2 & 0.2 & 0.2 & 0.2 & 0.2 \\
\hline 31 & LOGICA MATEMATICA & 0.2 & 0.2 & 0.2 & 0.2 & 0.2 \\
\hline 32 & MATEMATICAS DISCRETAS & 0.2 & 0.2 & 0.2 & 0.2 & 0.2 \\
\hline 33 & METODOLOGIA DE LA INVESTIGACION & 0.2 & 0.2 & 0.2 & 0.2 & 0.2 \\
\hline 34 & METODOS NUMERICOS & 0.2 & 0.2 & 0.2 & 0.2 & 0.2 \\
\hline 35 & PRIOGRAMACION ORIENTADA A OB.JETOS & 0.2 & 0.3 & 0.2 & 0.3 & 0 \\
\hline 36 & PROTOCOLOS DE COMUNICACIÓN DE DATOS & 0.1 & 0.1 & 0.1 & 0.3 & 0.4 \\
\hline 37 & REDES & 0.1 & 0.1 & 0.1 & 0.1 & 0.6 \\
\hline 38 & SISTEMAS DE INFORMACION & 0.4 & 0.3 & 0.1 & 0.1 & 0.1 \\
\hline 39 & SISTEMAS DIGITALES & 0.2 & 0.2 & 0.2 & 0.2 & 0.2 \\
\hline 40 & SISTEMAS OPERATIVOS & 0 & 0.3 & 0.3 & 0.3 & 0.1 \\
\hline 41 & SISTEMAS OPERATIVOS PARA RED & 0.1 & 0.1 & 0.1 & 0.4 & 0.3 \\
\hline 42 & TEMAS SELECTOS DE SISTEMAS & 0.2 & 0.2 & 0.2 & 0.2 & 0.2 \\
\hline 43 & ADMINISTRACION DE CENTROS DE COMPUTO & 0.2 & 0.2 & 0.2 & 0.2 & 0.2 \\
\hline 44 & INTERCONEXIONY SEGURIDAD EN REDES & 0.1 & 0.1 & 0.1 & 0.1 & 0.6 \\
\hline 45 & RESIDENCIAS PROFESIONALES & 0.2 & 0.2 & 0.2 & 0.2 & 0.2 \\
\hline 46 & INTELIGENCIA ARTIFICIAL & 0.3 & 0.3 & 0.1 & 0.2 & 0.1 \\
\hline 47 & SEMINARIO DE TITULACION & 0.2 & 0.2 & 0.2 & 0.2 & 0.2 \\
\hline 48 & PLANEACION ESTRATEGICA & 0.2 & 0.2 & 0.2 & 0.2 & 0.2 \\
\hline 49 & SISTEMAS DE INSTRUMENTACIONY CONTROL & 0.1 & 0.1 & 0.3 & 0.2 & 0.3 \\
\hline 50 & SISTEMAS ELECTRONICOS DE COMUNICACIÓN & 0.1 & 0.1 & 0.3 & 0.1 & 0.4 \\
\hline 51 & TEORIA DE CONTROL & 0.2 & 0.1 & 0.3 & 0.2 & 0.2 \\
\hline 52 & TRANSMISIONY COMUNICACIÓN DE DATOS & 0.2 & 0.1 & 0.1 & 0.1 & 0.5 \\
\hline 53 & SISTEMAS EXPERTOS & 0.3 & 0.3 & 0.1 & 0.2 & 0.1 \\
\hline
\end{tabular}


Modelo difuso para evaluar la pertinencia de un programa educativo de nivel superior

Tabla 6. Resultado de VPA para el PE en ISC.

\begin{tabular}{cccccc}
\hline \multicolumn{5}{c}{ Nombre de áreas } \\
\hline VPA & SSCAE & NTISC & DHSAAE & AHSAE & RCNE \\
\%VPA & 10.9 & 11 & 9.8 & 10.5 & 10.8 \\
\hline
\end{tabular}

Al aplicar el Factor de Ganancia $(\boldsymbol{F G})$ por años de experiencia, el cual está determinado en la ecuación 6 se tiene como resultado la Tabla 7.

Tabla 7. $F \boldsymbol{G}$ para el PE en ISC.

\begin{tabular}{cccccc}
\hline \multicolumn{5}{c}{ ÁREA EGEL-ICOMPU } \\
\hline $\boldsymbol{F} \boldsymbol{G}$ & SSCAE & NTISC & DHSAAE & AHSAE & RCNE \\
$\boldsymbol{F G}_{\text {Alto }}$ & 0.6307 & 0.625 & 0.7015 & 0.6547 & 0.6365 \\
$\boldsymbol{F G}_{\text {Medio }}$ & 0.5045 & 0.5 & 0.5612 & 0.5238 & 0.5092 \\
$\boldsymbol{F G}_{\text {Bajo }}$ & 0.2522 & 0.25 & 0.2806 & 0.2619 & 0.2546 \\
\hline
\end{tabular}

De acuerdo a la ecuación 8, se generaron 25 reglas difusas, distribuidas como sigue: 10 para el TDSS, 5 para el TDS y 10 para el ANS.

\subsection{Análisis de resultados}

Entre 2014 y marzo 2015, 26 egresados del PE de ISC plan flexible realizaron el EGEL-ICOMPU, a los cuales se les aplicó el modelo difuso propuesto, al realizar un análisis de los resultados obtenidos, se tiene la Tabla 8 , la cual considera el puntaje pronosticado en cada área del EGEL-ICOMPU comparado con el puntaje real obtenido por los sustentantes en dichas áreas.

En la Tabla 8, se observa lo siguiente:

- El área DHSAAE, es la de menor puntaje, tanto en el pronóstico como en el puntaje obtenido, esto tiene sentido, toda vez que en el cálculo del $\boldsymbol{V P A}$ se muestra que es el área de menor impacto en el EGEL, es importante realizar acciones que lleven a elevar este puntaje.

- En las cuatro áreas restantes, se observa que en promedio los sustentantes alcanzan más de 1000 puntos, por lo que se alcanzaría un TD, sin embargo, en ninguno de los casos se visualiza un puntaje mayor a 1040, lo que implica, que se corre el riesgo de obtener un ANS.

- En promedio se tiene una aproximación del $96.28 \%$, lo cual representa un buen grado de aproximación. 
Tabla 8. Comparativo del puntaje pronosticado vs puntaje obtenido .

\begin{tabular}{|c|c|c|c|c|c|c|c|c|c|c|c|}
\hline \multirow{2}{*}{ No. } & \multicolumn{5}{|c|}{ Puntaje pronosticado en cada área del EGEL- } & \multicolumn{5}{|c|}{ Puntaje obtenido por el sustentante en cada área } & \multirow{2}{*}{ APROXIMACIÓN } \\
\hline & SSCAE & NTISC & DHSAAE & AHSAE & RCNE & SSCAE & NTISC & DHSAAE & AHSAE & RCNE & \\
\hline 1 & 1148 & 1153 & 1015 & 1140 & 944 & 1146 & 1178 & 1049 & 1175 & 915 & $97.65 \%$ \\
\hline 2 & 918 & 922 & 903 & 917 & 917 & 920 & 947 & 898 & 937 & 829 & $96.75 \%$ \\
\hline 3 & 924 & 927 & 909 & 920 & 920 & 961 & 885 & 898 & 894 & 851 & $95.80 \%$ \\
\hline 4 & 930 & 935 & 920 & 930 & 937 & 824 & 947 & 898 & 786 & 915 & $92.51 \%$ \\
\hline 5 & 932 & 936 & 917 & 928 & 930 & 934 & 957 & 898 & 1024 & 937 & $97.11 \%$ \\
\hline 6 & 1078 & 1081 & 915 & 929 & 934 & 1056 & 1043 & 920 & 916 & 958 & $97.95 \%$ \\
\hline 7 & 944 & 947 & 1043 & 1064 & 941 & 947 & 978 & 1008 & 1047 & 958 & $97.94 \%$ \\
\hline 8 & 1054 & 932 & 914 & 1046 & 926 & 1021 & 957 & 986 & 1024 & 980 & $95.86 \%$ \\
\hline 9 & 1063 & 938 & 923 & 1054 & 939 & 1003 & 998 & 898 & 1047 & 980 & $96.08 \%$ \\
\hline 10 & 944 & 1080 & 927 & 940 & 1075 & 988 & 1058 & 942 & 980 & 1001 & $96.10 \%$ \\
\hline 11 & 1058 & 935 & 919 & 927 & 1066 & 1003 & 957 & 898 & 851 & 1020 & $95.26 \%$ \\
\hline 12 & 1062 & 1084 & 922 & 933 & 1061 & 1074 & 1149 & 942 & 980 & 1075 & $97.04 \%$ \\
\hline 13 & 925 & 929 & 909 & 921 & 1064 & 947 & 998 & 898 & 980 & 1094 & $96.18 \%$ \\
\hline 14 & 1055 & 932 & 913 & 1061 & 1064 & 1003 & 998 & 964 & 1137 & 1113 & $94.38 \%$ \\
\hline 15 & 1067 & 928 & 912 & 921 & 1071 & 1039 & 988 & 920 & 959 & 1131 & $96.25 \%$ \\
\hline 16 & 1062 & 1069 & 920 & 1055 & 1058 & 1003 & 1074 & 898 & 1069 & 1020 & $97.21 \%$ \\
\hline 17 & 1018 & 1129 & 1006 & 1017 & 1018 & 1056 & 1159 & 1090 & 1024 & 1057 & $96.37 \%$ \\
\hline 18 & 1031 & 1035 & 1018 & 1030 & 1030 & 1056 & 1058 & 1042 & 1047 & 1057 & $97.86 \%$ \\
\hline 19 & 1025 & 1029 & 1010 & 1022 & 1021 & 1021 & 998 & 1028 & 1024 & 1094 & $97.57 \%$ \\
\hline 20 & 1057 & 936 & 1028 & 1067 & 1070 & 1021 & 947 & 1070 & 1137 & 1094 & $96.64 \%$ \\
\hline 21 & 1097 & 1100 & 945 & 1093 & 1169 & 1003 & 1059 & 942 & 1024 & 1113 & $94.90 \%$ \\
\hline 22 & 1132 & 1137 & 1095 & 1122 & 1126 & 1146 & 1149 & 1090 & 1024 & 1113 & $97.28 \%$ \\
\hline 23 & 1072 & 1090 & 1045 & 1068 & 1105 & 1021 & 1104 & 1070 & 959 & 1131 & $95.54 \%$ \\
\hline 24 & 1104 & 1110 & 1070 & 1096 & 1093 & 1198 & 1134 & 1070 & 1175 & 1020 & $95.23 \%$ \\
\hline 25 & 1125 & 1130 & 1090 & 1115 & 1117 & 1198 & 1134 & 1049 & 1114 & 1150 & $97.34 \%$ \\
\hline \multirow[t]{2}{*}{26} & 1105 & 1113 & 1077 & 1096 & 1107 & 1185 & 1149 & 1171 & 1175 & 1150 & $94.36 \%$ \\
\hline & 1035.77 & 1020.65 & 971.73 & 1015.85 & 1027.04 & 1029.77 & 1038.58 & 982.19 & 1019.58 & 1029.08 & $96.28 \%$ \\
\hline
\end{tabular}

La evaluación de pertinencia a través del modelo difuso aplicado para el PE en ISC, ha permitido detectar lo siguiente:

- El área de Nuevas Tecnologías para la Implementación de Sistemas de Cómputo, es la de mayor presencia en el EGEL-ICOMPU, esto es, que el sustentante tiene las competencias para seleccionar y aplicar los principios y metodologías de desarrollo necesarios en la generación de nueva tecnología en la implementación de sistemas de cómputo.

- El área de Desarrollo de Hardware y su Software Asociado para Aplicaciones Específicas, es la de menor presencia para el PE ISC, lo que implica que el sustentante no tiene las competencias para analizar distintos problemas y proponer soluciones. Se sugiere fortalecer dicha área, adecuando los contenidos del PE en ISC, para que ello redunde en un mejor aprovechamiento de los alumnos y por ende mayores puntuaciones en el EGEL-ICOMPU.

- Los sustentantes con mayor experiencia en el campo laboral, son lo que han alcanzado un TD.

Dada la generalidad de este modelo, puede ser aplicado a cualquier PE de una IES, para dar respuesta a las siguientes preguntas: ¿Qué áreas de las que evalúa el EGEL tienen mayor o menor impacto en un PE?, ¿Qué grado de impacto tiene cada UDA en un PE con respecto a las áreas del EGEL?, ¿Los factores de tiempo de egreso y experiencia profesional impactan sobre un TD?

\section{Conclusiones}

La lógica difusa está enfocada en la toma de decisiones cuando existen datos o conocimientos inciertos, existiendo diversas aplicaciones para la vida real y donde se 
reemplaza al humano por un sistema difuso basado en reglas. En este trabajo, se eligió esta debido a que ofrece varias ventajas, descritas como sigue:

- Al momento de dar un pronóstico, proporciona una manera sencilla y eficaz para extraer conclusiones de vaguedad, ambigua o información imprecisa. Por lo tanto, simula la toma de decisiones y puede trabajar a partir de datos aproximados para obtener soluciones precisas.

- Incorpora una forma alternativa de pensar, lo que permite que todo pronóstico sea modelado con un nivel de abstracción que refleje conocimiento y experiencia a partir de reglas de inferencia.

- Permite expresar conocimiento con conceptos subjetivos, tales como los resultados que pueden ser obtenidos en el EGEL: ANS, TDS y TDSS, los cuales pueden ser mapeados de manera exacta dentro de los rangos difusos.

- Es un método eficiente que rápidamente proporciona uno o más pronósticos como soluciones.

- Ofrece varios beneficios, tales como el rendimiento, simplicidad, bajo costo y productividad en el modelado de un sistema de pronóstico difuso.

El estudio aquí planteado ayudará, entre otros aspectos, a conocer si existe pertinencia entre los Programas de Estudios de las IES y las áreas que evalúa el EGELCENEVAL; los resultados obtenidos, proporcionarán información útil a las autoridades de los espacios académico para tomar decisiones que coadyuven a alcanzar un Testimonio de Desempeño, abordando con ello el problema de la baja Eficiencia de titulación que existe actualmente.

\section{Referencias}

1. SEP.: Programa Sectorial de Educación 2013-2018. http://www.sep.gob.mx/work/models/sep1/Resource/4479/4/images/PROGRAMA _SECTORIAL_DE_EDUCACION_2013_2018_WEB.pdf (2015)

2. UAEM.: Curriculum de Ingeniería en Sistemas y Comunciaciones. http://dep.uaemex.mx/curricular/sitio_/index.php (2015)

3. Tamayo, M.: El proceso de la investigación cientifica. Limusa, pp. 37-45 (2009)

4. CENEVAL.: Guía para el sustentante: Examen General para el Egreso de la Licenciatura en Ingeniería Computacional. http://archivos.ceneval.edu.mx/archivos_portal/20882/GuiadelEGEL-COMPU.pdf (2015)

5. Trejo, R.: Experiencia de desempeño en el examen EGEL de los egresados de la Carrera de Ingeniería Química del Instituto Tecnológico de Aguascalientes, Conciencia Tecnológica, pp. 41-43 (2008)

6. Izar, J., López, H.: El puntaje que obienen los estudiantes de contaduría pública en el examen general de egreo de la licenciatura (EGEL) y su relación con el promedio obtenido durante su carrera. Hitos de Ciencias Económica Administrativa, pp. 2734 (2010)

7. Bravo, O., Rodríguez, J.: El Examen General de Egreso de Licenciatura de la Facultad de Lenguas extranjeras: Una experiencia en proceso. 
http://fel.uqroo.mx/adminfile/files/memorias/Articulos_Mem_FONAEL_II/Bravo _Gomez_Omar_\&_Rodriguez_Reyes_Jose_Miguel.pdf. (2013)

8. Vazquez, J., Bastidas, B., Hirales, M., Botello, R., Ramírez, A.: La evaluación de egresados de licenciatura, una perspectiva comparada entre los resultados obtenidos en dos versiones del EGEL-CENEVAL. http://www.saece.org.ar/docs/ congreso4/trab45.pdf. (2015)

9. León, Y., Palacios, G., Martínez, J., Juárez, F.: Experiencia del Instituto Tecnológico de Pachuca en los procesos de evaluación-EGEL, Sistemas y Gestión Educativa, pp. 1016-1021 (2015)

10. Zadeh, L.: Fuzzy sets, Information and Control. pp. 338-353 (1965)

11. Dubois, D., Prade, H.: Fuzzy sets and systems: Theory and Applications. Academic Press (1980)

12. Kosko, B.: Fuzzy systems as universal approximators. IEEE, pp. 1329-1333 (1994)

13. Mandani, E.: Application of fuzzy logic to approximate reasoning using linguistic synthesis. IEEE (1977)

14. IEC.: International Electrotechnical Commission. http://www.iec.ch/dyn/www/f?p=103:91:0::::FSP_LANG_ID:25?q=Fuzzy Control Language (2014) 